\section{Born free}

\section{Roger F. Searle}

The Immunology of Human Pregnancy. By Henry N. Claman. Humana: 1993. Pp. 232. $\$ 59.50$.

THE birth of the topic of reproductive immunology is widely acknowledged to stem from a seminal paper by the late Sir Peter Medawar in 1953, in which he recognized and addressed the immunological problems presented by the evolution of viviparity in vertebrates. Important advances in cellular immunology and molecular biology, especially in the past 10 years, have largely clarified the immunological paradox of pregnancy, but not yet definitively explained how the 'foreign' fetus survives to term without provoking maternal immune rejection. The intellectual challenges posed by the maternal-fetal immunological relationship are now particularly relevant: issues such as HIV infection in pregnancy, and the development of immune approaches to birth control and fertility regulation, attract much attention. Recently, this rapidly moving and multifaceted subject has been the focus of several comprehensive state-of-the-art texts such as Immunology of Pregnancy edited by G. Chaouat (CRC, 1993) and The Immune System in Disease edited by G. M. Stirrat and J. R. Scott (Baillière Tindall, 1992). It is against this background that Henry Claman's review has to be judged.

Claman sets out to explore in depth fundamental questions, but readers expecting such will be left unfulfilled and let down. The book has been written very much with the busy clinician in mind and conveys only the most superficial impression, an approach that is less than satisfactory for the serious researcher or specialist. It is therefore curious that although the author's original impetus for writing was his involvement in the controversial treatment of

New Journals Issue
This year, Nature's annual new journals review
supplement will appear in the issue of 7
October. Publishers and learned societies are
invited to submit journals for review, taking
note of the following criteria:
Journals that first appeared during or after
June 1991 and issued at least four separate
numbers by the end of April 1993 will be
considered.
Frequency of publication must be at least
three times a year. The main language used
must be English.
Deadline for submission is the end of May.
When submitting journals for review, please
send at least four different issues (the first, the
most recent and any two others) of each title.
For further information telephone Peter
Tallack on $071-836-6633$ (011-44-71-836-
6633 from the US).

recurrent spontaneous miscarriage by immunotherapy, he has largely ignored the topical clinical arena. This is a considerable shortcoming. Disappointingly for a book published in 1993, there are few references more recent than 1991 (and very few 1991 references at that).

On the credit side, the text is highly readable and will easily guide the nonspecialist clinician through the complexities of fundamental immunology and provide a useful introduction to the immunology of human pregnancy. The format of the book follows predictable well-tried themes, covering immunological aspects of the placenta, maternal immunocompetence during pregnancy and maternal responses to fetal antigens. The style is relaxed and informative.

Topics of current intense interest such as the expression and regulation of HLA-G class I molecules on trophoblast cell populations at the maternal-fetal interface are mentioned, but inevitably provide a picture from around early 1991. The implications of HLA-G ex- pression for the outcome of pregnancy are given scant attention and "remain to be explored", which today is simply not true. Similarly, statements such as "the study of cytokines in the placenta is just starting" are nonsensical in light of the rapid advances highlighting the crucial regulatory role that growth factors play in reproductive processes. The chapters on immune disorders of pregnancy and recurrent pregnancy loss, however, are especially informative and Claman is here to be congratulated for summarizing succintly a large body of data in a balanced and objective way.

The attraction of the book is that it gives clinicians an easily readable albeit superficial overview that will serve as a useful introduction: and even specialists in the field are likely to find useful information in one or more of the chapters. $\square$

Roger F. Searle is in the Department of Immunology, The Medical School, University of Newcastle upon Tyne, Newcastle upon Tyne NE2 4HH, UK.

\title{
Molecular receptors in the round
}

\section{Leonard F. Lindoy}

Macrocyclic Chemistry: Aspects of Organic and Inorganic Supramolecular Chemistry. By B. Dietrich, P. Viout and J.-M. Lehn. VCH: 1993. Pp. 384. DM148, £61, \$99.

MACROCYCLIC ligands - cyclic organic molecules with a central cavity - are used by nature to bind metal ions in such important molecules as haem, chlorophyll and vitamin $B_{12}$. Several natural antibiotics are also macrocyclic and work by metal binding. There are good reasons for nature to choose macrocyclic species to bind metal ions - foremost among these is that the binding is usually considerably stronger than for comparable noncyclic ligands.

In addition to natural rings, a lot of synthetic macrocyclic molecules have been investigated over the past 30 years. Many can act as receptors for small molecules as well as different ion types (including anions). Ionic and molecular recognition is a frequent characteristic of such host-guest interactions, aided by a cavity in the host with fixed or semi-fixed binding sites. Host-guest assemblies of this type fall in the realm of supramolecular chemistry the chemistry of synthetic receptors and large molecular assemblies that often mimic nature's chemistry. Supramolecular chemistry is now a large and expanding field that is serving to underwrite an emerging new age in chemistry: the age of molecular devices.
In 1987, the Nobel prize for chemistry was awarded to Charles Pedersen, Donald Cram and Jean-Marie Lehn for their pivotal contributions to macrocyclic and supramolecular chemistry. This book is based on a series of lectures on macrocyclic chemistry delivered by Professor Lehn at the Collège de France some years ago. It is divided into two sections: "Macrocycle synthesis" and "Macrocyclic Complexes - Cryptates".

The first section contains a valuable discussion of synthetic approaches and general strategies for obtaining macrocyclic ligands. In keeping with the French group's formidable record in synthetic macrocyclic chemistry, the content of this section is wide ranging, covering both practical and theoretical aspects. The remaining section focuses on the complexes of both natural and synthetic macrocyclic molecules and presents a panoramic view. Included is an extended discussion of the chemistry of macrobicyclic ligands (the cryptands) and their complexes (the cryptates).

Overall, the book provides an effective way for researchers and graduate students to gain a foundation in the fascinating concepts of the field. It supplies the background for appreciating the many exciting advances currently unfolding.

Leonard F. Lindoy is in the Department of Chemistry and Biochemistry, James Cook University, Townsville 4811, Queensland, Australia.

NATURE · VOL 363 - 6 MAY 1993 